\title{
AGN From the Perspective of New Approaches in Astrophysics
}

\author{
S.G. Iskudarian \\ 378433, Aragatsotn, Byurakan Observatory, Byurakan, Armenia.
}

\begin{abstract}
In all probability, AGN are one of the most convincing observable gleams of the Superforce in the Universe.
\end{abstract}

What are the new approaches in astrophysics? How do we look at AGN from the perspective of these new approaches (Iskudarian 2000a)? This postertext is connected with the sixth point of Iskudarian (2000a) - with the idea about similar behavior of galaxies and elementary particles (Iskudarian 2000b). Some physicists think that the Universe is ruled by a Superforce (Davies 1985). We don't see the last one, but if we want to see it, we must be ready to resist the great disaster. Such a huge disaster was the Big-Bang in the Universe.

It is not news if I say that there is something that is impossible on Earth, but in the Universe it is common. For example, there are the decay of the proton and the existence of the magnetic monopole. They are the very mechanism which I offer as the explanation of the huge energies in AGN. The image of NGC5128 (Sandage 1964) is very much like the decay of a proton, only on macro scales. And the fact that the first AGN were Seyferts in giant spirals (Suchkov 1988) allows us to think that here will be the participation - the function of the monopole also. And so, from the perspective of new approaches in astrophysics, we look at the AGN not only as agents which carried the huge energies of the Big-Bang to the corners of the observable Universe (Iskudarian 1998a), but also as large-scale elementary particles - conglomerates of protogalactic matter with the "genes" of elementary particles (Iskudarian 1998a). The activity of the nuclei of galaxies according to Ambartsumian's theory $(1964,1966)$ is just the activity of protogalactic matter with the combination of the processes of decay, explosion and ejection of some portions of protogalactic matter and gas. These processes are accompanied by the eruption of huge energies. According to theoretical physicists, only such energies can provide the decay of the proton or monopole (Davies 1985, Okun 1984). They have elaborated this part of their theory in such detail and so deeply, that one can say with great confidence that AGN are of largescale merge display of the aforementioned two particles. Such a supposition we made on the basis of the following facts:1.The first galaxies with active nuclei were Seyfert galaxies and as a rule, they were giant spiral galaxies (Suchkov 1988), 2.By the Byurakan ideas (Ambartsumian 1958, Iskudarian 1975), there is an intimate connection between the mechanism of formation of galaxies and the method of origin of their individual structural pecularities 3.Two worlds of the Universe submit to the one general regularity, which is the ejection of the first type stellar population from the entrails of the second type one(in protogalactic stage, of course (Iskudarian 1996,1997)), 4. The separate physical groups of galaxies with AGN, in all probability, are different 
evolutionary stages of the same objects (B.V.Komberg,preprint),5.M87 itself with its wide environment(look at Fig.1 from Iskudarian (1993), where the distribution of the brightest and faintest members of the N94 and N106 rich groups of galaxies (Huchra \& Geller 1987) is given by their 1 and b coordinates) is a huge monopole with its fields of forces. These fields of forces are:1.The jet of M87, with blue condensations in it,2.The net of the rich globular cluster population (Iskudarian 1993),3.Elliptical galaxies around M87 (Fig.Ic of Iskudarian 1993), 4.Two symmetric details(in Fig.1c they are in contour line). It is interesting that these details have the same content of galaxies, only with contrary arrangement.

The whole picture with its fields of forces is only reminiscent of a huge monopole, but not in outward appearance. Theoretical physicists suppose that a monopole looks like an onion. The distribution of the faintest members of N106 looks more like an onion (look at Fig.1b of Iskudarian 1993). The last one is one more field of forces.

In fact, on outward appearance, in the center of the "monopole" (M87 with its extended environment) there is a proton (the nucleus of M87), which suffered decay, maybe not just once. As the groups N94 and N106 are connected physicaly, one may think that the huge monopole around M87 is physically connected with a closed looplike superstring (Iskudarian 1998b) to the nucleus of M87. The author admits that such a supposition is very bold. She is not a physicist, but she asks theoretical physicists to pay attention to this problem, especially to the part where they say that the monopole plays the role of catalyst in the process of the decay of the proton.

\section{References}

Ambartsumian, V.A., 1958, XI Solvay Conference, Brussels.

Ambartsumian, V.A., 1964, XIII Solvay Conference, Brussels.

Ambartsumian, V.A., 1966, IAU Symposium N29 Byurakan.

Davies, P., 1985, Superforce, New York.

Huchra, J.P. \& Geller, M.J.,1987, ApJS, 52, 61.

Iskudarian, S.G., 1975, Byur.obs.Contr.,46, 73.

Iskudarian, S.G., 1993, International Workshop on"Galaxy Clusters and Large Scale Structures in the Universe", Sesto Pusteria (Bolzano,Italy).

Iskudarian, S.G., 1996, International workshop on "Hubble deep fields", Baltimore, USA.

Iskudarian, S.G., 1997, Meeting Astro-4, Moscow.

Iskudarian, S.G., 1998a, IAU Symposium N194, Byurakan.

Iskudarian, S.G., 1998b, Euroconference,"The Evolution of Galaxies on Cosmological Timescales", Tenerife, Spain.

Iskudarian, S.G., 2000a, JENAM-2000, Moscow.

Iskudarian, S.G., 2000b, Internatl. workshop on "Hubble deep fields", Muenchen.

Okun, L.B., 1984, Physics of Elementary Particles, Science, Moscow.

Sandage, A.R., 1961, The Hubble Atlas of Galaxies, Washington.

Suchkov, A.A., 1988, Science, Moscow. 